\title{
Actividades lúdicas para fortalecer el aprendizaje multidisciplinar y el acercamiento intercultural
}

\author{
Pedro Verdejo $^{a}$, Lucia Hilario ${ }^{b}$, Ana Abalos ${ }^{c}$ \\ ${ }^{a}$ Universidad CEU-Cardenal Herrera (UCH), Valencia, España, pverdejo@uchceu.es. ${ }^{b}$ UCH, \\ luciah@uchceu.es. ${ }^{\mathrm{c}} \mathrm{UCH}$, ana.abalos@uchceu.es.
}

\begin{abstract}
Resumen
El profundo cambio experimentado en estos últimos años tras la internacionalización de los estudios en la Escuela de Arquitectura en la Universidad CEU-Cardenal Herrera, ha conllevado la llegada de alumnos con una gran diversidad geográfica y cultural, planteando la necesidad de apostar por un profundo cambio y revisión de las metodologías y planteamientos docentes.
\end{abstract}

Pero más allá de adaptar el canal, medio y contexto al nuevo tipo de alumno sin diluir el mensaje, se ha apreciado un nuevo problema de adaptación, donde la gran diferencia entre sus culturas y tradiciones, hace que se aprecien nuevas barreras para su integración.

Con este fin, se planteó una actividad lúdica que integrara contenidos de las asignaturas de Matemáticas II, Dibujo Arquitectónico II e Introducción a la Arquitectura. Para ello se tomó como excusa la temática de las Fallas, algo puramente autóctono pero que ha permitido mediante el conocimiento de la tradición y cultura local que los nuevos alumnos compartieran sus experiencias y culturas propias.

La experiencia ha permitido comprobar que no es necesario excesivos recursos materiales y humanos para fortalecer el aprendizaje de contenidos, la correlación entre asignaturas y favorecer la integración del nuevo alumnado internacional, y como no, acercarlos también a nuestra fiesta más internacional.

Palabras clave: Internacionalización, actividad lúdica, transversalidad. 


\section{Introducción}

\subsection{Antecedentes}

La internacionalización de los estudiantes en la Escuela de Arquitectura del CEU y la llegada de alumnos desde diferentes partes del mundo, ha producido un cambio drástico en el alumno respecto al que estábamos acostumbrados.

Por tanto, nos encontramos en un nuevo contexto donde los alumnos de nuevo ingreso, provienen de realidades docentes con metodologías de aprendizaje muy diversas, y con una gran disparidad de costumbres y hábitos dependiendo de cuál sea su procedencia. Desde que se inició este proceso, la Escuela Superior de Enseñanzas Técnicas se encuentra inmersa en el desarrollo de nuevas metodologías docentes adaptadas al nuevo perfil del alumnado, con asignaturas más activas y favoreciendo el enlace entre contenidos.

Por otro lado, la gran diversidad de culturas y tradiciones han conllevado en cierto modo, a una mayor dificultad a la hora de la integración del grupo, generando una problemática particular.

Nuestra inquietud ante esta problemática, más allá de seguir con la dinámica de adecuar el estilo de enseñanza al estilo de aprendizaje del alumno, ha motivado el planteamiento de una actividad que fomentara la convivencia y relación entre nuestros estudiantes, aprendiendo de la cultura más cercana a su nuevo entorno, sin menospreciar la oportunidad para seguir integrando contenidos y evidenciar la transversalidad de las asignaturas, pero en este caso, bajo un contexto lúdico, distendido y divertido.

\subsection{Objetivos}

Los objetivos del presente proyecto se encuentran enmarcados dentro de la estrategia de adecuación del estilo de enseñanza al estilo de aprendizaje del nuevo alumno internacional, facilitando para ello, el aprendizaje mediante la innovación y creatividad didáctica, en este caso, mediante una actividad lúdica. Para ello se establecen tres ámbitos de objetivos.

- Por un lado unos objetivos académicos, donde se sigue enmarcando en el diseño de actividades que vinculen contenidos de diferentes asignaturas de primer curso de Fundamentos de Arquitectura, comprobando la aplicabilidad y transversalidad de los contenidos aprendidos.

- La segunda línea se enmarcaría en un ámbito social, donde se pretende que los nuevos alumnos internacionales, de procedencia, culturas y costumbres muy dispares, realicen una actividad grupal, fomentando la relación entre iguales y compartiendo experiencias comunes. Se pretende reforzar así la visión del conjunto como grupo, promoviendo su integración. 
- El último rango de objetivos se posicionará en el ámbito cultural. Como son alumnos extranjeros que van a vivir un periodo de tiempo en nuestra ciudad, es importante que conozcan y entiendan nuestra cultura, en este caso, promoviendo una actividad dentro de una de nuestras fiestas más tradicionales: las Fallas.

\subsection{Actuaciones y experiencias previas}

El planteamiento inicial de esta actividad fue propuesto en el curso anterior, como una actividad mucho más autónoma consistente en una visita durante el montaje de los monumentos de las Fallas. En esta acción ya participaron los profesores de las asignaturas implicadas, permitiendo decidir in situ las acciones más adecuadas para vincular los contenidos de las asignaturas a la actividad.

Una vez comprobado el interés y la aceptación que provocó entre los alumnos, nos pareció un contexto perfecto para generar una actividad conjunta que permitiera afrontar unos objetivos más ambiciosos de forma más organizada, y en coordinación con el calendario lectivo.

\section{Descripción de la actividad}

Dado que el presente proyecto de innovación no se trata de un estudio al uso, sino que muestra una experiencia educativa, se incluirá en este apartado la metodología utilizada así como el diseño de la actividad.

\subsection{Metodología}

La metodología propone implicar al menos a tres asignaturas por semestre y lograr que, desde todas ellas exista un vínculo común con la arquitectura valenciana o de la cultura de la Fallas. En esta actividad han participado las asignaturas de Mathematics II, Architecture Drawing II y Introduction to Architecture, con un total de 18 alumnos.

Aunque la actividad no supone la preparación específica de contenidos en cada materia, sí que exige la adaptación en la explicación de sus conceptos, la presentación de ejemplos o incluso la realización de alguna práctica vinculadas con el tema propuesto.

Posteriormente, se procederá a la realización de una visita, donde el alumno podrá comprobar la incidencia de los conocimientos aprendidos en el hecho construido. Para ello 
se propone una visita enmarcada en tres acciones: apreciar la arquitectura, descubrir las costumbres y reflexionar sobre el trabajo del arquitecto.

Finalmente, se pretende realizar una reflexión sobre la visita y los contenidos aprendidos. Para ello se propone una nueva acción en clase donde los alumnos expondrán las fotos realizadas, explicando a sus compañeros sus reflexiones. Las fotos quedarán recogidas posteriormente en una exposición en el hall de la Escuela.

\subsection{Diseño de la actividad}

La actividad está planteada para desarrollarse en tres fases, diferenciadas tanto en el tipo de acciones como en su temporalidad.

En la primera de ellas, constará de la impartición de conocimientos propios de las asignaturas implicadas en la actividad, pero orientando algunos de los conceptos explicados a ejemplos que puedan tomarse de la arquitectura valenciana o de la cultura de la Fallas.

- En la asignatura de Introducción a la Arquitectura, se abordarán conceptos como el color, la luz, la composición o el programa arquitectónico, abordando una serie de ejemplos propios de la arquitectura de la ciudad de Valencia. En las prácticas desarrolladas en esta asignatura, se realizarán pequeñas maquetas y bocetos, como herramienta de trabajo propia del arquitecto.

- En la asignatura de Matemáticas II, el estudio de las geometrías básicas tomará ejemplos de monumentos falleros de años anteriores, pudiendo apreciar leyes de crecimiento y operaciones booleanas. Además, en los edificios visitados observamos la importancia de los conceptos geométricos estudiados en la asignatura de Matemáticas y su aplicación al mundo de la arquitectura.

En la asignatura de Dibujo Arquitectónico II, mediante el estudio de la representación gráfica, se han tomado ejemplos de arquitectura valenciana para mediante el dibujo, proceder a su estudio, análisis e interpretación, bajo las leyes propias de la representación arquitectónica. (Figura 1) 

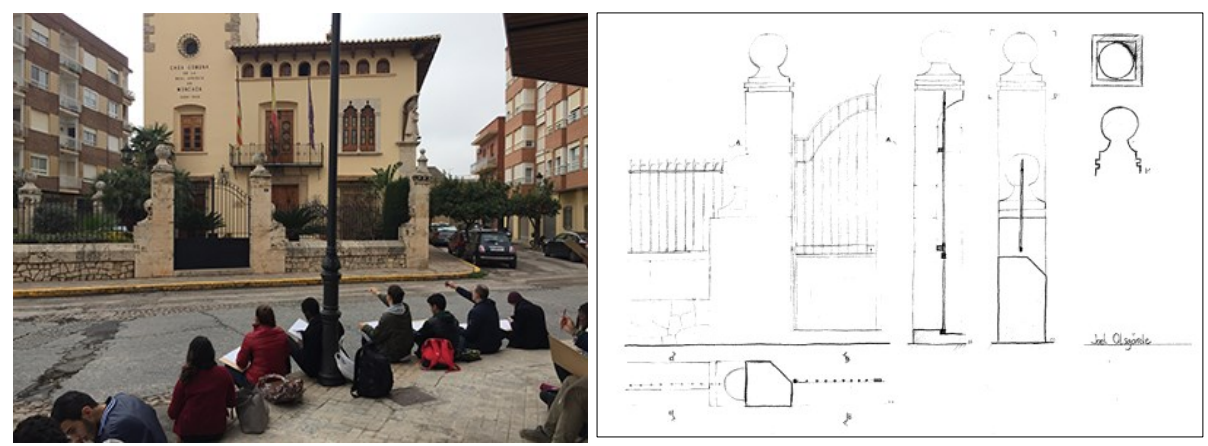

Fig. 1. Ejemplos de trabajo de los alumnos con edificios históricos, Fuente: autores

Estos ejemplos pasarán a convertirse en ejercicios de la asignatura, por lo que no supondrán una mayor carga lectiva, sino una reorientación de las prácticas propuestas. El análisis y representación de los elementos arquitectónicos permite reflexionar sobre su geometría, proporción y ornamentación, aprendiendo de forma indirecta la relación entre el dibujo y el hecho arquitectónico. En este apartado se inicia también la relación entre los contenidos de Dibujo Arquitectónico II e Introducción a la Arquitectura.

La segunda fase tiene por objetivo realizar una visita por la ciudad de Valencia, para poder apreciar la aplicación real de los contenidos aprendidos. Aunque la salida tiene una duración de un solo día, se desarrollan tres acciones:

Acción 1: Durante la mañana se ha procedido a realizar una serie de visitas a diversos edificios y espacios arquitectónicos representativos de la ciudad. Entre los espacios visitados estarían: el Palacio de Colomina. (Explicación del estilo y composición utilizada en el palacio, recorriendo sus diferentes espacios interiores para apreciar su composición geométrica), Baños del Almirante. (aunque de dimensiones reducidas, permite apreciar el efecto de la luz en el espacio arquitectónico y la geometría de sus espacios, bóvedas y lucernarios), la Almoina, (visita interior para apreciar las pinturas y policromías interiores, el espacio arquitectónico, su importancia en la historia de la ciudad de Valencia), visita a la parroquia de San Nicolás, comprobando sus impresionantes bóvedas policromías.

Acción 2: Visita al montaje de las fallas de Almirante Cadarso y Conde Altea, de la mano del artista fallero Luís Pascual, del equipo de Manolo Algarra, ganador del "ninot indultat" en las últimas cuatro ediciones. Nos explicó los pormenores del montaje de los monumentos, su construcción, la estructura que las sustenta, acabados y sentido del monumento. Los alumnos pudieron observar de primera mano el desarrollo de los trabajos. (Figura 2) 
Acción 3: Visita al estudio de arquitectura "obrador abalosllopis" para poder ver como es el trabajo profesional del arquitecto y que materiales utiliza. Es importante recalcar que los alumnos pudieron apreciar desde la organización diaria del trabajo de un estudio de arquitectura, los proyectos en los que se encuentran inmersos o más importante aún, diferentes técnicas de trabajo como el uso de maquetas previas para la ideación del proyecto arquitectónico, metodología análoga a la seguida en clase para la realización de ejercicios de análisis en la asignatura de Introducción a la Arquitectura.
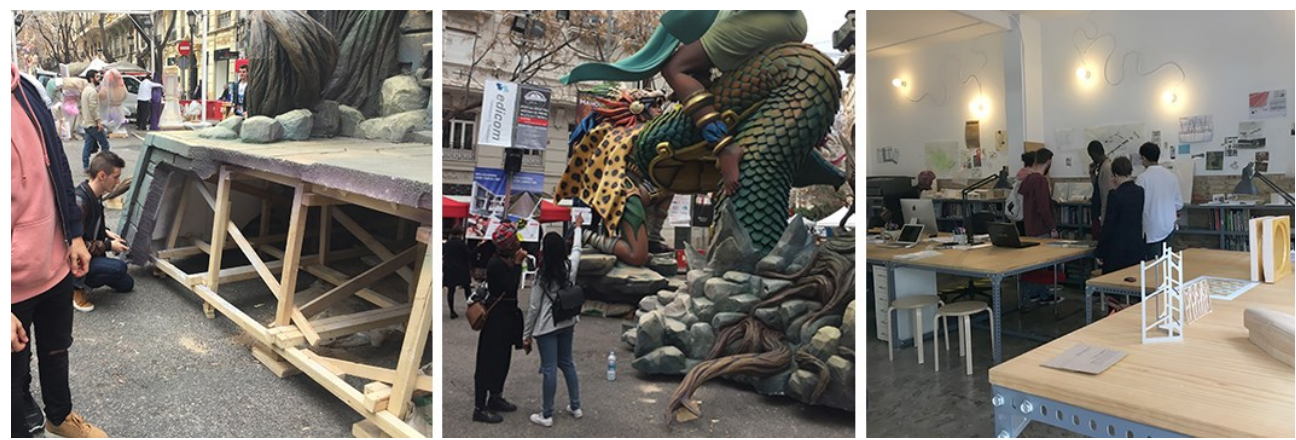

Fig. 2. Vistas de las diferentes acciones llevadas a cabo en las visitas, Fuente: autores

Por último, en una última fase se ha pretendido comprobar el cumplimiento de los objetivos. Para ello se han propuesto diferentes actividades dependiendo de la asignatura, que van desde la recogida de imágenes de arquitectura histórica y contemporánea durante las visitas en Dibujo Arquitectónico II, a la aplicación de herramientas proyectuales como la realización de maquetas o la utilización de ejemplos visitados para la explicación de contenidos de la asignatura de Introducción de la Arquitectura, o al reconocimiento de superficies geométricas estudiada en la asignatura de Matemáticas. (Figura 3)
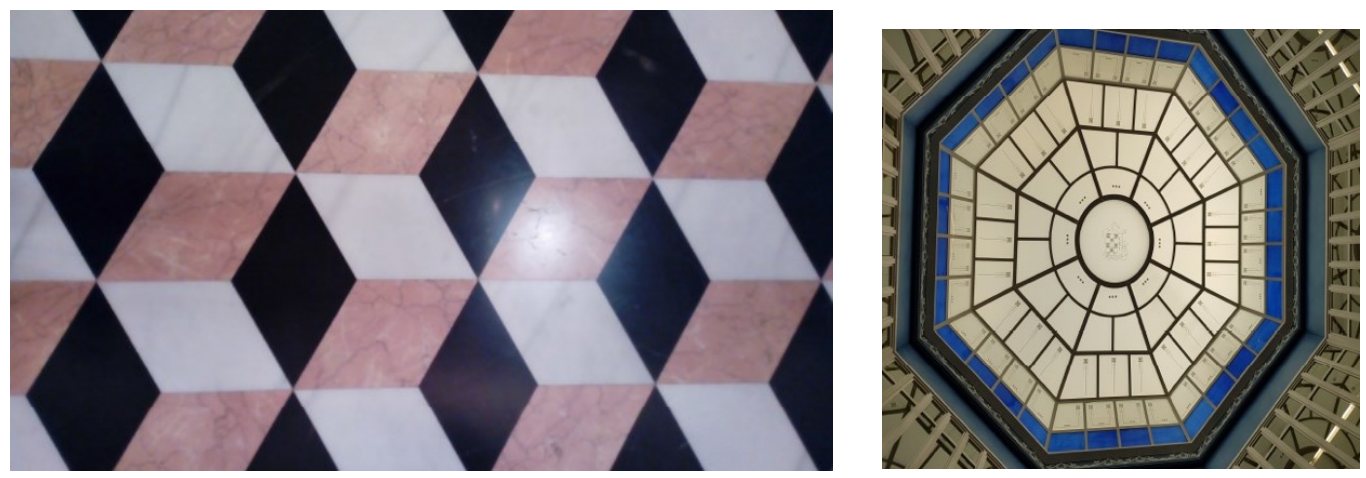

Fig. 3. Ejemplos de geometrías y patrones geométricos encontrados en los edificios visitados. Fuente: autores 
Se realizó una última acción que consistió en una exposición de las fotografías del grupo, con la intención de que cada alumno internacional muestre desde su punto de vista particular, lo que más le sorprendió de la tradición de las Fallas.

\subsection{Planificación temporal}

Se ha implantado la actividad en tres fases diferentes:

Fase 1: Impartición de docencia: durante el mes mes de febrero y primera quincena de marzo en el horario lectivo de cada asignatura.

Fase 2: Visitas

Acción 1: Visita a centro histórico de Valencia. Mañana.

Acción 2: Visita al montaje de una Falla. Tarde.

Acción 3: Visita a un despacho de arquitectura en Valencia. Tarde.

Fase 3: Puesta en común y reflexión sobre contenidos. (abril, mayo)

Acción 4: Exposición fotográfica: (mayo)

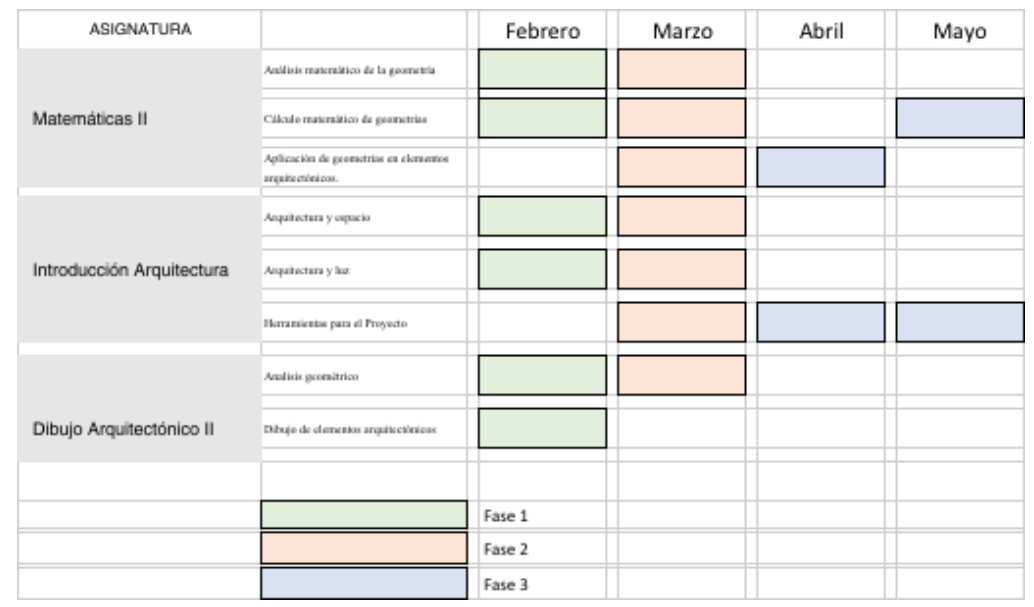

Fig. 4. Cronograma de asignaturas implicadas y acciones, Fuente: autores

\section{Resultados}

La actividad ha sido desarrollada por un total de 18 alumnos de primer curso de Fundamentos de Arquitectura, con nacionalidades tan dispares como Francia, Ruanda, Finlandia, Rusia, India, Mozambique, Zambia, Alemania, Siria, Líbano, Suecia, Albania, Reino Unido, etc. 
Los resultados, al igual que los objetivos planteados, se pueden desglosar en tres ámbitos:

Respecto al ámbito cultural, se aprecian los resultados más inmediatos, ya que la actividad lúdica de la visita por Valencia, ha permitido fomentar el conocimiento del nuevo entorno del alumno internacional, en cuanto a las costumbres y cultura locales.

Por lo que se refiere al ámbito social, la actividad ha mejorado y favorecido el establecimiento de relaciones entre los alumnos internacionales, así como un mayor acercamiento e interacción entre los estudiantes y profesores, al desarrollar actividades más allá del marco de las aulas.

En cuanto al ámbito docente, se han podido establecer relaciones entre contenidos aplicables a la actividad, donde los alumnos han podido comprobar su utilidad y aplicabilidad, sobrepasando los límites teóricos de la asignatura.

Además, la visita a un despacho profesional permitió observar de primera mano la metodología proyectual y herramientas empleadas, comprobando la analogía con las utilizadas en las asignaturas proyectuales, lo que pone en valor las metodologías de aprendizaje utilizadas.

Por otro lado, la realización de prácticas relacionadas con la actividad pero sin suponer un incremento de la carga lectiva, ha sido apreciada favorablemente por los alumnos, incrementando su motivación y predisposición a participar en la actividad.

La realización de una encuesta de satisfacción final, ha mostrado que los alumnos quedaron ampliamente satisfechos en todos los aspectos de la actividad, justificando la conveniencia de la actividad. (anexo II)

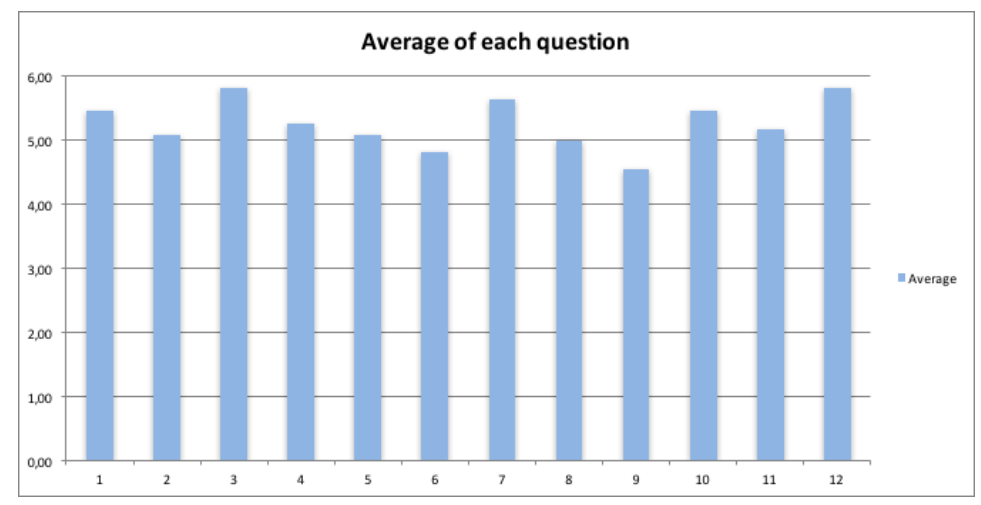

Fig. 5. Gráfico de satisfacción del alumnado. Fuente: autores 


\section{Conclusiones}

Por todo lo expuesto con anterioridad, consideramos que la realización de esta actividad ha fomentado en primer lugar la integración intercultural del nuevo alumno, favoreciendo la adaptación a su entorno cultural más cercano.

La innovación con actividades creativas y didácticas fuera del límite físico del aula, favorece la motivación y participación activa del alumnado, reforzando el aprendizaje y asimilación de los contenidos sin entrar en conflicto con el resto de metodologías de aprendizaje.

La utilización de dinámicas lúdicas no implica un consumo alto de recursos económicos y personales, ni incrementa la carga lectiva del alumno, únicamente plantea una reorganización de las actividades y prácticas de cada asignatura, por lo que queda confirmada la aplicabilidad del proyecto.

Los alumnos han mostrado una amplia satisfacción por su participación en la actividad, por lo que habiendo sido demostrada que es factible su aplicabilidad, debería continuarse en el futuro como parte de la dinámica de aprendizaje.

\section{Referencias}

De la cruz, A. (1999): Formación del profesor universitario en metodología docente”,en Aprender y enseñar en la Universidad. Iniciación a la docencia universitaria. Jaén, Universidad de Jaén.

Fernández M. A. (2010). La evaluación orientada al aprendizaje en un modelo de formación por competencias en la educación universitaria. Revista de Docencia Universitaria, vol. 8, p. 11-34.

Gargallo L. B., Pérez P. C. (2014), Transversal Competences for Employment and Profile of Excellent University Students, Procedia - Social and Behavioral Sciences, Vol. 139, pg. 305-313.

Marcelo, C. (2001). Función docente: nuevas demandas para viejos propósitos. Madrid. Síntesis.

Zabalza, M. A. (2000): La enseñanza universitaria: el escenario y los protagonistas. Madrid, Narcea. 


\section{ANEXO I}

\section{ENCUESTA DE SATISFACCIÓN}

ACTIVITY: Las Fallas as a recreational activity to strengthen multidisciplinary and intercultural learning.

The rating scale is from 1 (lowest value: worst) to 6 (highest value: best):

(1) Strongly disagree. (2) Very disagree. (3) I disagree. (4) All right. (5) I agree. (6) I absolutely agree.

\begin{tabular}{|c|c|c|c|c|c|c|c|}
\hline 1 & The activity has been properly organised. & 1 & 2 & 3 & 4 & 5 & 6 \\
\hline 2 & I think I found the activity useful for my training & 1 & 2 & 3 & 4 & 5 & 6 \\
\hline 3 & I have felt in a comfortable environment & 1 & 2 & 3 & 4 & 5 & 6 \\
\hline 4 & $\begin{array}{l}\text { I have found it rewarding to share the activity with colleagues of } \\
\text { other nationalities }\end{array}$ & 1 & 2 & 3 & 4 & 5 & 6 \\
\hline 5 & $\begin{array}{l}\text { I have known architectural spaces in the history of Valencia that I } \\
\text { may never have visited on my own. }\end{array}$ & 1 & 2 & 3 & 4 & 5 & 6 \\
\hline 6 & $\begin{array}{l}\text { I have been able to verify some of the knowledge of Theory of } \\
\text { Architecture in the activity. }\end{array}$ & 1 & 2 & 3 & 4 & 5 & 6 \\
\hline 7 & I have managed to understand the tradition of Fallas monuments & 1 & 2 & 3 & 4 & 5 & 6 \\
\hline 8 & $\begin{array}{l}\text { I have been able to appreciate the mathematical geometries in the } \\
\text { different spaces visited. }\end{array}$ & 1 & 2 & 3 & 4 & 5 & 6 \\
\hline 9 & $\begin{array}{l}\text { I think it has helped my learning by seeing how geometry is } \\
\text { applied in architecture. }\end{array}$ & 1 & 2 & 3 & 4 & 5 & 6 \\
\hline 10 & $\begin{array}{l}\text { It has been useful for me to be able to see what it is like to work in } \\
\text { a professional architectural office }\end{array}$ & 1 & 2 & 3 & 4 & 5 & 6 \\
\hline 11 & I think the activity has been adequate for my first year. & 1 & 2 & 3 & 4 & 5 & 6 \\
\hline 12 & $\begin{array}{l}\text { I would repeat the same kind of activity another year. } \\
\text { (with a different theme) }\end{array}$ & 1 & 2 & 3 & 4 & 5 & 6 \\
\hline
\end{tabular}

